\title{
Performance Analysis of Hierarchical and Flat Network Routing Protocols in Wireless Sensor Network Using Ns-2
}

\author{
Anupam Jamatia, Kunal Chakma, Nirmalya Kar, Dwijen Rudrapal, and Swapan Debbarmai
}

\begin{abstract}
In the era of technological advancement wireless communication using different advance technology and sensor networks hold the promise of facilitating large-scale and realtime data processing in complex environments like defense service specially Military service, Medical service, disaster management, wildlife monitoring and precision agriculture to habitat monitoring and logistics applications etc. Sensor networks have the characteristics of fault tolerance and rapid development make them very promising in the above mentioned field. The main objective of this paper is to analyze the performance of two categories routing protocols i.e. Hierarchical and Flat network routing protocols in Wireless Sensor Networks (WSN). For simulation purpose we have taken mainly Temporally-Ordered Routing Algorithm (TORA), Low Energy Adaptive Clustering Hierarchy (LEACH), and INtrusion-tolerant routing protocol for wireless SEnsor NetworkS (INSENS) protocols.
\end{abstract}

Index Terms-Hierarchical routing protocol, flat routing protocol, WSN, TORA, leach, INSENS.

\section{INTRODUCTION}

A wireless sensor network represents a new wireless communicating paradigm of many self-organized autonomous small size low-power, low-cost sensor nodes that are used to co-operatively monitor complex physical or environmental conditions, such as motion, temperature, sound etc. Such sensors are generally deployed in indoor and outdoor scenarios [1]. These sensors nodes are communicate with each other to share data and information to monitor a specific environment. A sensor node generally composed of sensor, processor, transceiver, and power units. In addition to these functionalities a sensor nodes also has the capability of routing. Due to the remote nature of WSNs deployment, sensor nodes face energy optimization and quick routing discovery problems [2]. To address these issues different routing techniques have been proposed. One of the networks routing protocol is Low Energy Adaptive Clustering Hierarchy (LEACH) which has clustering mechanism falls under the hierarchical network routing. The difference between Mobile ad-hoc Networks (MANET) and Wireless Sensor Networks (WSN) is that collecting information is done by WSN with higher number of nodes deployed once in their lifetime while the other one is designed for distributed computing in ad-hoc manner [3]-[7].

\section{Routing PRotocols IN WSN}

There are various routing protocols shown in the Fig. 1

Manuscript received July 1, 2014; revised October 12, 2014.

The authors are with the National Institute of Technology, Agartala 799055 India (e-mail: anupamjamatia@gmail.com). has proposed for routing data in wireless sensor networks they have resource management capability, deployment capability etc. There are few distinct routing protocols that are based on quality of service awareness or network flow whereas all other routing protocols can be classified as hierarchical or location based and data centric. To ensure proper routing of authentic data WSN protocol has different techniques.

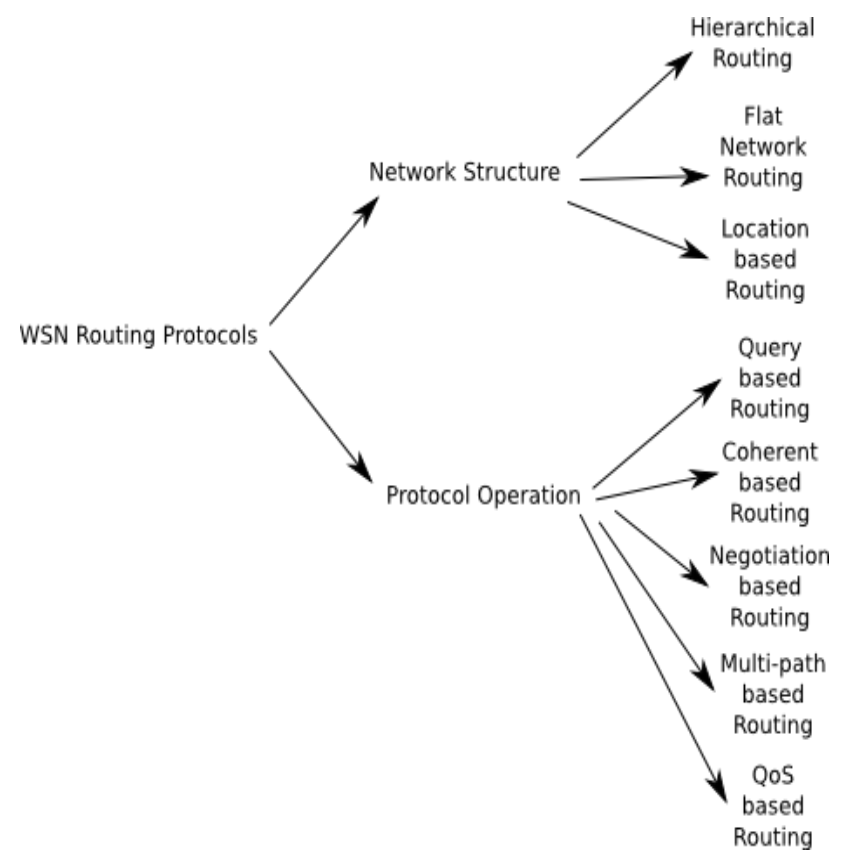

Fig. 1. Different network architecture and routing techniques.

In this paper we have mainly work on two network structure i.e. hierarchical routing and flat routing. LEACH and INSENS falls under the category of hierarchical routing and TORA falls under the category of flat routing.

\section{A. $L E A C H$}

It is based on energy efficient, hierarchical cluster-based routing protocols for sensor networks. The basic principle is that it assigns overall energy consumption of the network uniformly to each sensor node through periodically selecting different nodes as cluster-head. This makes the survival time of nodes close to the lifetime of network [8]. Thus, the energy consumption can be reduced and the lifetime of the entire network can be prolonged. LEACH functionality divided into two main steps: the set-up phase and the steadystate phase with different multiple access technologies to form clusters with non-cluster sensor nodes. This protocol is designed to enable maximum energy by enabling nodes to enter the sleep state. This protocol also assumes that all nodes are energy-limited and introduced the idea of rotating 
the cluster-head position among all the nodes in the network [9], [10].

\section{B. INSENS}

Deng, Han, Mishra [11] designed and implemented a secure and Intrusion-tolerant routing protocol for wireless Sensor NetworkS (INSENS). This protocol constructs forwarding table at each node to facilitate communication between base station and the sensor nodes. It minimizes computation, communication, storage, and bandwidth requirements at the sensor nodes at the expense of increased computation, communication, storage, and bandwidth requirements at the base station. INSENS does not rely on detecting intrusions, but rather tolerates intrusions by bypassing the malicious nodes. An important property of INSENS is that while a malicious node may be able to compromise a small number of nodes in its vicinity, it cannot cause widespread damage in the network. Redundant multipath routing improves intrusion tolerance by bypassing malicious nodes. INSENS operates correctly in the presence of (undetected) intruders. An important property of INSENS is that while a malicious node may be able to compromise a small number of nodes in its vicinity, it cannot cause widespread damage in the network [11], [12].

\section{TORA}

In the Wireless Sensor Networks The TemporallyOrdered Routing Algorithm (TORA) falls under the category of flat routing. It is fully distributed routing protocol for multihop networks which has unique approach for packet routing. In TORA, each mobile node is assigned a temporal-order sequence number to support multi-path routing from a source to a specific destination node [13]. As the main property of ad-hoc routing protocol there should not be any centralized control. TORA is basically a hybrid routing protocol, a mixture of proactive and reactive ad-hoc routing protocol. This protocol is basically designed to minimize the communication overhead associated with adapting to network topological changes [14], [15]. Rather than routing optimally TORA allows its operation to be biased towards bandwidth conservation and high reactivity.

\section{Simulation Result AND ANALYsis}

To analyze the objective of this paper we have used discreate event simulator ns- 2 which is a powerful tool for simulating ad-hoc networks in linux platform i.e. ubuntu 12.04 LTS. But to simulate WSN in ns-2, it needs to have additional module to represent the protocols specific to WSN. Mannasim is a framework for WSN simulation based on ns-2. It extends ns-2 by introducing new modules for design, development and analysis of different WSN applications. The goal of Mannasim is to develop a detailed simulation framework, which can accurately model different sensor nodes and applications while providing a versatile test bed for algorithms and protocols [16].

In this simulation, the traffic sources are CBR (continuous-bit-rate). The traffic type was connectionless UDP. The data packet size is 512 bytes. Speed of the nodes is $8 \mathrm{kbps}$. The mobility model uses random waypoint model in a rectangular filed of $600 \mathrm{~m} \times 500 \mathrm{~m}$ with variable nodes $50,75,100,125$, and 150 . In this mobility model, each sensor node starts its journey from a fixed chosen location to a fixed chosen destination. Once the destination is reached it stop. From starting point to destination it chose its way randomly, after a pause time it goes ahead to destination. Different network scenario for different numbers of node, pause time and speeds are generated. Total simulation time is 100 seconds.

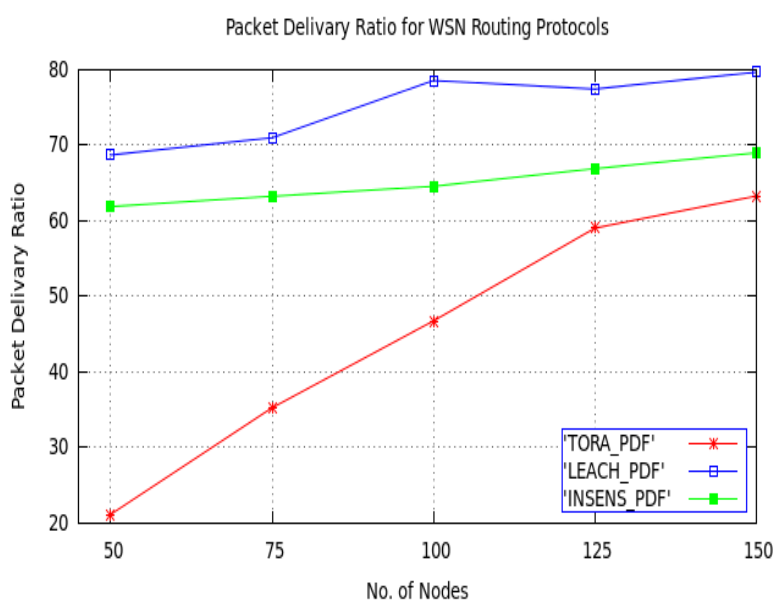

Fig. 2. Packet delivery ratio for WSN routing protocols.

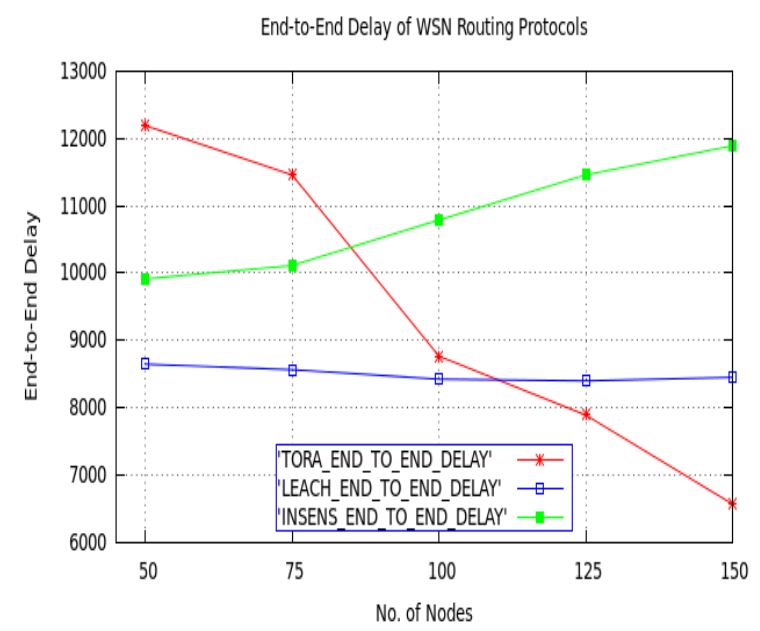

Fig. 3. End-to-end delay for WSN routing protocols.

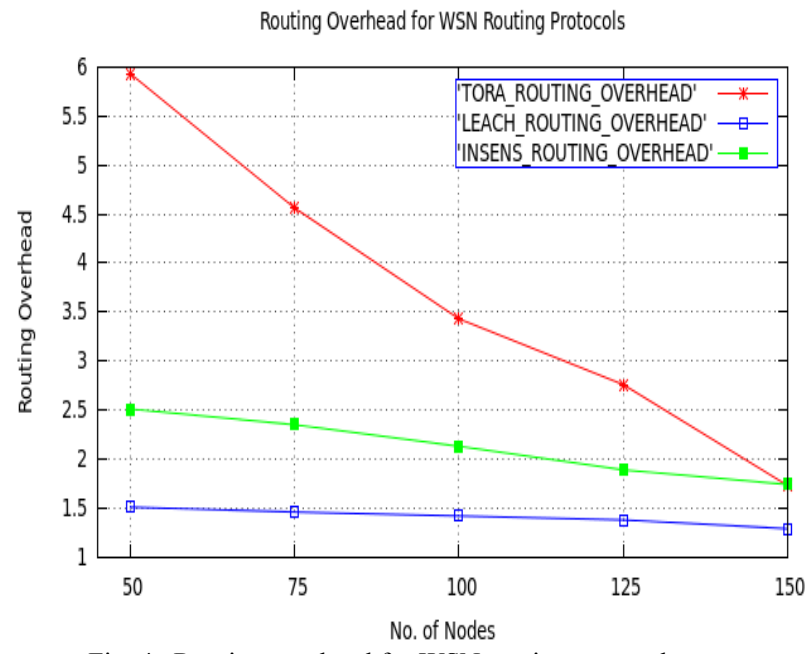

Fig. 4. Routing overhead for WSN routing protocols.

\section{A. Performance Metrics}

We choose the three following performance metrics to analyze the performances of three WSN protocols. The 
following performance metrics are considered for evaluation:

Packet Delivery Fraction (PDF): The percentage ratio of the data packets delivered to the destinations to those generated by the sources usually by the traffic generator.

Throughput: The ratio of the data packets delivered to the destinations to those generated by the source.

Normalized Routing Load (NRL): The number of routing packets "transmitted" per data packet "delivered" at the destination.

\section{B. Result Analysis of Simulation}

The simulation results based on the performance metrics are shown in the following sub section with figures. Figures shows comparison between the three protocols by varying different numbers of nodes on the basis of the abovementioned metrics as a function of drop rate, received, send, time and speed.

\section{1) Packet delivery ratio}

The packet delivery ratio denotes the percentage ratio between the number of packets that are received and the number of packets sent by the traffic generator. TORA has a lower packet delivery ratio than the other two protocols, as TORA reduces communication overhead; it increases unnecessary overhead due to its route adaptation feature in response to topological changes in the scenario of the network. From the Fig. 2 it has been shows that TORA produces fewer throughputs due to extra overhead of the destination or path establishment and for upgrading the path in an adaptive fashion. LEACH performs better as it forms cluster heads that reduce overhead. INSENS sends same packets multiple times to the destination thus lowering the packet delivery ratio.

\section{2) Average end to end delay}

This delay includes processing and queuing delay in each intermediate node i.e. the time elapsed until a demanded route is available. In this case unsuccessful route establishments are ignored. It includes all possible delays caused by queuing at the interface queue, retransmission delays at the MAC, and propagation and transfer times. By localization, TORA tries to minimize communication overhead. This decreases end-to-end delay. But LEACH has a lower end to end delay as LEACH is a single-hop clustering routing protocol. Aggregates data from a cluster head. The Average end to end delay is higher for INSENS as in this protocol all sensor nodes share authentication key with the base station creating more delay shows in the Fig. 3.

\section{3) Routing load}

Routing load measures the scalability of the protocols, how much overhead a protocol can take. The routing overhead measures by the total number of control packets sent divided by the number of data packets sent successfully. As mentioned before TORA produces higher control load due to its adaptive nature. Other protocols need to re-initiate a route discovery when a link fails. TORA would be able to patch itself up around the point of failure. This feature allows TORA to scale up to larger networks but has higher overhead for smaller networks. INSENS sends more packets than the other protocols, and the difference increases with increasing numbers of nodes in the network. This difference is attributed to the overhead involved in dealing with security and intrusion-tolerance issues. LEACH performs better even with the routing load of forming clusters heads, as the area of the routing load is divided between the different clusters. There is also a co relation with the no. of nodes. LEACH and INSENS perform better with higher number of nodes shows in the Fig. 4.

\section{CONCLUSIONS}

Comparing all three routing protocols, it is seen that TORA performs less than the other two protocols. But TORA itself improves its performance when the node number increases. Other protocols need to re-initiate a route discovery when a link fails. TORA would be able to patch itself up around the point of failure. This feature allows TORA to scale up to larger networks but has higher overhead for smaller networks. LEACH has a better performance overall than the other two protocols, having a single-hop cluster based architecture. The idea of employing cluster-heads does quite help to give a higher Packet Delivery Ratio (PDR).For INSENS, as quality of service is the main issue, consequently the performance of the network was slightly degraded, although there seems to be a close competition between INSENS and LEACH, as a better QoS again means a higher PDR. A huge number of WSN protocols are available having varied network architecture and operation, each suited best for a specific environment, e.g.-WAR (Wireless Anonymous Routing), Phantom routing, SPINS etc. Comparison between these protocols can be done with additional parameters such as random node mobility, increased number of nodes etc. to determine if they can tolerate harsh and changing environments, which the protocols are prone to specially when used outdoors, and find out which one performs best in a particular environment.

\section{REFERENCES}

[1] D. Varagnolo, P. Chen, L. Schenato, and S. S. Sastry, "Performance analysis of different routing protocols in wireless sensor networks for real-time estimation," in Proc. Decision and Control, 2008. CDC 2008. 47th IEEE Conference, 2008, pp.4904-4909.

[2] K. Latif, M. Jaffar, N. Javaid, M. N. Saqib, U. Qasim, and Z. A. Khan, "Performance analysis of hierarchical routing protocols in wireless sensor networks," in Proc. the Broadband, Wireless Computing, Communication and Applications (BWCCA), 2012 Seventh International Conference, 2012, pp. 620-625.

[3] M. Pandey and S. Verma, "Performance evaluation of AODV for different mobility conditions in WSN," in Proc. the Multimedia, Signal Processing and Communication Technologies (IMPACT), 2011 IEEE International Conference, 2011, pp. 240-243.

[4] A. Badi, I. Mahgoub, M. Slavik, and M. Ilyas, "Investigation of the effects of network density on the optimal number of clusters in hierarchical wireless sensor networks (WSNs)," High-Capacity Optical Networks and Enabling Technologies (HONET), 2010. pp. 171-177.

[5] M. Raza, G. Ahmed, N. M. Khan, M. Awais, and Q. Badar, "A comparative analysis of energy-aware routing protocols in wireless sensor networks," in Proc. Information and Communication Technologies (ICICT) International Conference, pp.1-5, 2011.

[6] M. Rajeswari, P. U. Maheswari, S. Bhuvaneshwari, and S. Gowri, "Performance analysis of AODV, DSR, TORA and OLSR to achieve group communication in MANET," in Proc. Advanced Computing (ICoAC), Fourth International Conference, pp. 1-8, 2012.

[7] Y. Hu, W. Li, and Z. H. Kang, "Study on energy efficient hierarchical routing protocals of wireless sensor network," in Proc. Information Engineering, 2009. ICIE '09. WASE International Conference, 2009, pp. 325-328.

[8] Y. X. Chan and Q. Zhao, "On the lifetime of wireless sensor networks," Communications Letters, vol. 9, no. 11, pp. 967-978, 2005. 
[9] C. M. Li, G. P. Tan, J. Y. Wu, Z. Zhang, and L. Z. Xu "Analyzing cluster-head selection mechanisms and improving the LEACH," in Proc. Electronics, Communications and Control (ICECC), International Conference, 2011, pp. 747-750.

[10] A. P. Renold, R. Poongothai, and R. Parthasarathy, "Performance analysis of LEACH with gray hole attack in wireless sensor networks," in Proc. 2012 International Conference on Computer Communication and Informatics (ICCCI), 2012, pp.1-4.

[11] J. Deng, R. Han, and S. Mishra, "INSENS: Intrusion-tolerant routing in wireless sensor networks," Technical Report CU_CS-939-02, Department of Computer Science, University of Colorado, November 2002.

[12] H. Dubois-Ferrière, D. Estrin, and M. Vetterli, "Packet combining in sensor networks," in Proc. the 3rd International Conference on Embedded Networked Sensor Systems, New York, NY, USA, 2005, pp. 102-115.

[13] Y. C. Tseng, M. S. Pan, and Y. Y. Tsai, "A distributed emergency navigation algorithm for wireless sensor networks," IEEE Computers, vol.39, no. 7, pp. 55-62, 2006.

[14] G. Spilios et al., "ZRP versus DSR and TORA: A comprehensive survey on ZRP performance," in Proc. 10th IEEE Conference on Emerging Technologies and Factory Automation, vol. 1, 2005, p. 1024.

[15] Z. W. Zhang and H. Zhou. "Empirical examination of mobile ad hoc routing protocols on wireless sensor networks," International Journal of Computer Networks and Communications, vol. 1, no. 1, pp. 75-87, 2009.

[16] J. G. Zhang, W. B. Li, D. X. Cui, X. L. Zhao, and Z. X. Yin "The NS2-based simulation and research on wireless sensor network route protocol," in Proc. 5th IEEE International Conference on Wireless Communications, Networking and Mobile Computing, 2009, pp. 1-4.

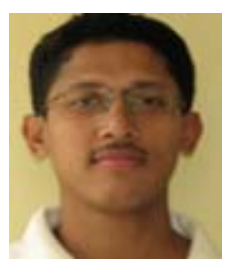

Anupam Jamatia is an assistant professor at the CSE Department of National Institute of Technology, Agartala, India. At present he is pursuing Ph.D. from NIT Agartala. His research interests include routing protocol in wireless networking and mobile computing and natural language processing.

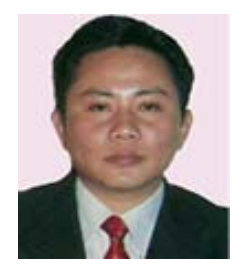

Kunal Chakma is an assistant professor in Computer Science \& Engineering Department at National Institute of Technology Agartala, Tripura, India. At present he is pursuing Ph.D. from NIT Agartala. His fields of interests are embedded systems, RTOS, networking, system software and distributed systems.

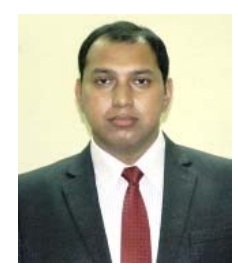

Nirmalya Kar is an assistant professor at CSE Dept. and Coordinator for C-DAC Centre of Excellence at NIT Agartala, India. He is a reviewer of various International Journals and also an editorial board member of the International Journal of Ambient Systems and Applications. At present he is pursuing $\mathrm{Ph}$.D. from NIT Agartala His research interest includes pervasive computing, cryptography \& RTOS.

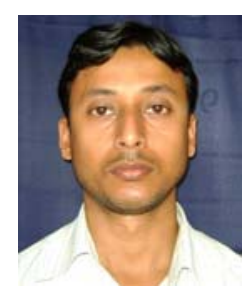

Dwijen Rudrapal is working as an assistant professor at the Computer Science and Engineering Department of National Institute of Technology, Agartala, India since 2006. He worked in industry for one year. At present he is pursuing Ph.D. from NIT Agartala Research. His research areas are biometric security and authentication.

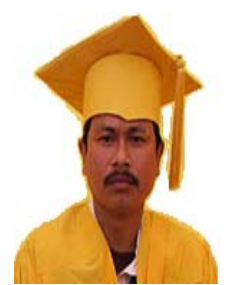

Swapan Debbarma is an assistant professor in Computer Science and Engineering Department at National Institute of Technology Agartala, Tripura, India. At present he is pursuing $\mathrm{Ph}$.D. from NIT Agartala in the area of nanotechnology. He also has interest in OS and networking. 